\title{
SCIENTIFIC REPORTS

\section{Hidden Hypocalcemia as a Risk Factor for Cardiovascular Events and All-Cause Mortality among Patients Undergoing Incident Hemodialysis}

\author{
Satoshi Yamaguchi ${ }^{1}$, Takayuki Hamano ${ }^{2,3^{*}}$, Yohei Doi ${ }^{1}$, Tatsufumi Oka ${ }^{1}$, Sachio Kajimoto ${ }^{1}$, \\ Keiichi Kubota ${ }^{1}$, SeiichiYasuda ${ }^{1}$, Karin Shimada ${ }^{1}$, Ayumi Matsumoto ${ }^{1}$, Nobuhiro Hashimoto ${ }^{1}$, \\ Yusuke Sakaguchi ${ }^{3}$, Isao Matsui ${ }^{1}$ \& Yoshitaka Isaka $^{1}$
}

Lower corrected calcium ( $\mathrm{cCa}$ ) levels are associated with a better prognosis among incident dialysis patients. However, cCa frequently overestimates ionized calcium (iCa) levels. The prognostic importance of the true calcium status defined by iCa remains to be revealed. We conducted a retrospective cohort study of incident hemodialysis patients. We collected data of iCa levels immediately before the first dialysis. We divided patients into three categories: apparent hypocalcemia (low iCa; $<1.15 \mathrm{mmol} / \mathrm{L}$ and low $\mathrm{CCa} ;<8.4 \mathrm{mg} / \mathrm{dL}$ ), hidden hypocalcemia (low iCa despite normal or high $\mathrm{cCa}$ ), and normocalcemia (normal iCa). The primary outcome was the composite of all-cause death and cardiovascular diseases after hospital discharge. Among the enrolled 332 patients, $75 \%$ of the patients showed true hypocalcemia, defined as $\mathrm{iCa}<1.15 \mathrm{mmol} / \mathrm{L}, 61 \%$ of whom showed hidden hypocalcemia. In multivariate Cox models including other potential risk factors, true hypocalcemia was a significant risk factor (hazard ratio [HR], 2.34; 95\% confidence interval $[\mathrm{Cl}], 1.03-5.34$ ), whereas hypocalcemia defined as corrected calcium $<8.4 \mathrm{mg} / \mathrm{dL}$ was not. Furthermore, hidden hypocalcemia was significantly associated with an increased risk of the outcome compared with normocalcemia (HR, 2.56; 95\% Cl, 1.11-5.94), while apparent hypocalcemia was not. Patients with hidden hypocalcemia were less likely to receive interventions to correct hypocalcemia, such as increased doses of active vitamin $\mathrm{D}$ or administration of calcium carbonate, than patients with apparent hypocalcemia (odds ratio, $0.45 ; 95 \% \mathrm{Cl}, 0.23-0.89$ ). Hidden hypocalcemia was a strong predictor of death and cardiovascular events, suggesting the importance of measuring iCa.

The inference of ionized calcium by Payne's formula ${ }^{1}$ frequently results in misclassifications of the calcium status $^{2-4}$. Over-correction of total calcium levels occurs easily, especially among patients with low serum albumin levels ${ }^{4}$. Patients with end stage renal disease tend to have low serum albumin levels ${ }^{5}$. Therefore, misclassifications of calcium status such as "hidden hypocalcemia: normal corrected calcium despite low ionized calcium" is expected to be prevalent among patients with chronic kidney disease (CKD) stage 5 . In fact, calcium misclassifications were reported among patients undergoing incident dialysis ${ }^{6}$.

Previous studies reported mixed findings regarding the associations between calcium and prognosis. A large cohort of maintenance hemodialysis patients showed a U-shaped association between corrected calcium and mortality $^{7}$. Two other studies reported that higher calcium levels were associated with worse prognosis among patients undergoing incident dialysis ${ }^{8,9}$. In a large cohort of patients with estimated glomerular filtration rate $(\mathrm{eGFR}) \geq 60 \mathrm{ml} / \mathrm{min} / 1.73 \mathrm{~m}^{2}$, a U-shaped association between calcium and mortality was observed ${ }^{10}$. Moreover,

${ }^{1}$ Department of Nephrology, Osaka University Graduate School of Medicine, Suita, Japan. ${ }^{2}$ Department of Nephrology, Nagoya City University Graduate School of Medical Sciences, Nagoya, Japan. ${ }^{3}$ Department of InterOrgan Communication Research in Kidney Disease, Osaka University Graduate School of Medicine, Suita, Japan. *email: hamatea@kid.med.osaka-u.ac.jp 
hypocalcemia was a risk factor for mortality in hospitalized patients with heart failure and CKD ${ }^{11}$. All these studies defined calcium status by corrected calcium levels. No clinical studies have investigated the prognostic implication of ionized calcium except for a study involving patients undergoing hemodialysis ${ }^{6}$.

Thus, we conducted a retrospective cohort study on patients just before the initiation of dialysis, in whom calcium misclassifications easily occur. The effect of pre-dialysis care of patients with CKD on the prognosis after the initiation of dialysis has received considerable attention in recent years ${ }^{8,12-15}$. The current study aimed to examine 1) the prevalence of hidden hypocalcemia just before the initiation of dialysis and 2) its prognostic implications after the initiation of dialysis.

\section{Methods}

Study design and populations. In this retrospective cohort study, we enrolled patients undergoing incident hemodialysis with ionized calcium measured between January 2008 and December 2016 in Osaka University Hospital. We excluded patients aged $\leq 20$ years, patients who started dialysis at the intensive care unit, and patients without data concerning albumin levels. This study was performed in accordance with the Helsinki Declaration. The Ethics Committee of Osaka University Hospital approved the study and waived informed consent based on the retrospective study design (approval number: 18026-2). We provided patients with the option to opt out of participation.

Data collection and laboratory measurements. We collected the latest data just before the initiation of dialysis. In the analysis, we used the data within 3 months prior to the initiation of dialysis. We measured ionized calcium with whole blood samples by using a blood gas analyzer (Siemens 348 and Radiometer ABL800 FLEX before and after November 2015, respectively) immediately after drawing the samples. We measured serum total calcium and albumin levels by the methyl xylenol blue method and the bromocresol purple method, respectively. We corrected serum total calcium levels by serum albumin levels if albumin levels $<4.0 \mathrm{~g} / \mathrm{dL}$ : corrected calcium $(\mathrm{mg} / \mathrm{dL})=\operatorname{calcium}(\mathrm{mg} / \mathrm{dL})+0.8 \times(4.0-\operatorname{albumin}[\mathrm{g} / \mathrm{dL}])^{16}$.

Outcomes. We followed up patients until August 2017. We obtained prognostic information from medical records or united questionnaires from dialysis facilities. The primary outcome was all-cause mortality and hospitalization for cardiovascular disease (CVD). CVD included myocardial infarction, unstable angina, heart failure, arrhythmia, hemorrhagic and non-hemorrhagic stroke, peripheral vascular events (including amputation), aneurysm dissection, or rupture. Patients were censored at the date of death or kidney transplant, or when they were lost to follow-up.

Statistical analyses. Corrected and ionized calcium was categorized as low $(<8.4 \mathrm{mg} / \mathrm{dL}$ and $<1.15 \mathrm{mmol} / \mathrm{L}$, respectively), normal $(8.4-10.0 \mathrm{mg} / \mathrm{dL}$ and $1.15-1.29 \mathrm{mmol} / \mathrm{L}$, respectively), and high $(>10.0 \mathrm{mg} /$ $\mathrm{dL}$ and $>1.29 \mathrm{mmol} / \mathrm{L}$, respectively $)^{17,18}$. We examined the prevalence of hypocalcemia defined by corrected calcium levels and ionized calcium levels and the risk factors for low ionized calcium levels using stepwise down logistic regression models. Most of the patients belonged to one of the following three categories: apparent hypocalcemia (low ionized calcium and low corrected calcium), hidden hypocalcemia (low ionized calcium despite normal or high corrected calcium), and normocalcemia (normal ionized calcium). We summarized baseline characteristics of these three groups. Data were presented as the number (percent) for categorical variables and as the mean (SD) for continuous variables with a normal distribution or the median (interquartile range) for those with a skewed distribution. We compared baseline characteristics between apparent and hidden hypocalcemia. The significance of differences in continuous variables between groups was tested using the Student's t test or Mann-Whitney test as appropriate. The difference in the distribution of categorical variables was tested using Fisher's exact test. We compared baseline characteristics between the three groups using analyses of variance, the Kruskal-Wallis tests, or Fisher's exact tests.

We examined the prognostic impact of hypocalcemia, defined by either ionized or corrected calcium, using the log-rank test, Kaplan-Meier curves, and Cox proportional hazards models. We also used these methods to examine whether the three calcium statuses (apparent hypocalcemia, hidden hypocalcemia, and normocalcemia) predicted the primary outcome after hospital discharge. We constructed several multivariable models: Model 1 adjusted for age, sex, eGFR, and history of diabetes; Model 2 adjusted for covariates in Model 1 plus covariates related to past history of CVD (percutaneous coronary intervention [PCI] or coronary artery bypass grafting [CABG], heart failure, pacemaker implantation, peripheral artery disease, and cerebrovascular infarction); Model 3 adjusted for covariates in Model 2 plus chronic kidney disease-mineral and bone disorder (CKD-MBD) parameters (phosphate, alkaline phosphates [ALP], and intact parathyroid hormone [iPTH]) and pH; and Model 4 adjusted for covariates in Model 3 plus nutrition or inflammation parameters (body mass index [BMI], albumin, and C-reactive protein [CRP]). In addition, we examined adjusted hazard ratios of each categories defined by ionized and corrected calcium status using model 4 with normal ionized and corrected calcium group as reference.

Furthermore, we examined associations between intervention to hypocalcemia and the primary outcome among patients with low ionized calcium levels using Cox proportional hazards models. A multivariate model was adjusted for age, sex, eGFR, and CKD-MBD parameters. Intervention to hypocalcemia was defined as either increased doses of vitamin D receptor activator (VDRA) during hospitalization or administration of calcium carbonate at discharge or both. Increased doses of VDRA included a switch from oral to intravenous VDRA. We compared the percentage of patients receiving intervention for hypocalcemia between apparent hypocalcemia and hidden hypocalcemia groups using the Fisher's exact test and logistic regression model. We also compared the percentage of patients receiving both increased doses of VDRA and administration of calcium carbonate at discharge. In these prognosis analyses, we excluded those who reached the outcome during hospitalization for dialysis initiation. 


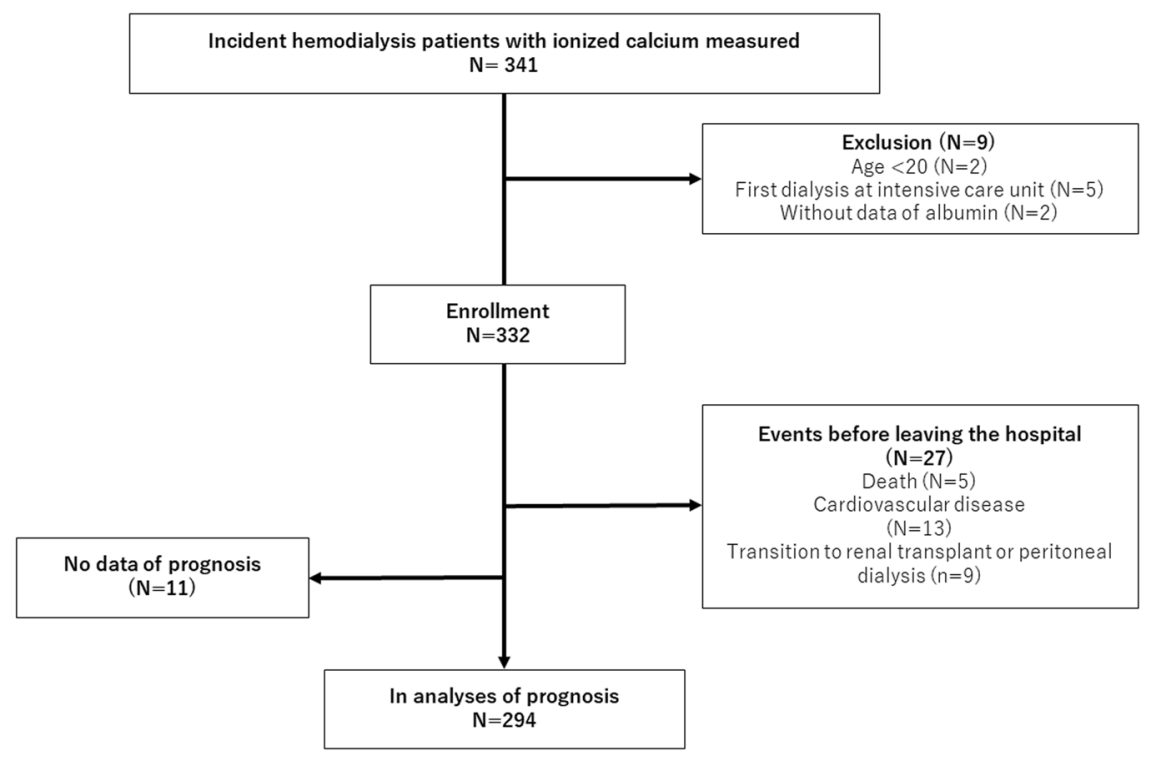

Figure 1. Flow diagram. In total, 341 patients were screened and 332 patients were enrolled ( 9 excluded). Among the enrolled patients, 18 patients reached the primary outcome and 9 patients withdrew from hemodialysis before leaving the hospital. Follow-up data after discharge were not obtained in 11 patients. These 38 patients were excluded in analyses of the association between calcium status before the initiation of dialysis and the primary outcome after leaving the hospital.

ALP, iPTH, and CRP levels were log-transformed to normalize the distribution. P-value $<0.05$ was considered statistically significant. All statistical analyses were conducted using Stata IC 14 statistical software (StataCorp, College station, TX, USA).

\section{Results}

Prevalence of hypocalcemia. Of the enrolled 332 patients (Fig. 1), (mean age, 64 years), 71\% were men: and the mean eGFR and serum albumin levels were $5.1 \mathrm{ml} / \mathrm{min} / 1.73 \mathrm{~m}^{2}$ and $3.0 \mathrm{~g} / \mathrm{dL}$, respectively. Their ionized calcium levels were measured immediately before the first dialysis session. The median duration between the first dialysis and the measurements of the other parameters was 0 day, except for iPTH, which was measured 1 day (median) before the first dialysis. Hypocalcemia, which was defined by low ionized calcium levels, accounted for $75 \%$ of the enrolled patients, whereas hypocalcemia, which was defined by low corrected calcium levels, accounted for $30 \%$. The significant risk factors for low ionized calcium levels were higher age, CRP, and iPTH; male sex; lower eGFR, BMI, albumin levels; and diabetes $(\mathrm{P}<0.05)$. Most of the patients $(98 \%)$ belonged to one of the following three categories: apparent hypocalcemia (29\%), hidden hypocalcemia (46\%), and normocalcemia (23\%) (Fig. 2). Patients with hidden hypocalcemia were likely to be older and had lower BMI and albumin levels, and higher $\mathrm{pH}$ than patients with apparent hypocalcemia, suggesting malnutrition $(\mathrm{P}<0.05)$. Patients with hidden hypocalcemia tended to have a history of diabetes mellitus, coronary artery disease, and heart failure $(\mathrm{P}<0.10$; not statistically significant) compared to patients with apparent hypocalcemia. Regarding the severity of hypocalcemia, patients with apparent hypocalcemia had lower ionized calcium levels than patients with hidden hypocalcemia $(\mathrm{P}<0.05)$ (Table 1). The prevalence of patients with ALP higher than the normal upper limit (>359 IU/L) was highest in the hidden hypocalcemia group (18\% vs. $10 \%$ in apparent hypocalcemia and $4 \%$ in normocalcemia, respectively: Fisher's exact test $\mathrm{P}<0.01$ ), despite them having lower iPTH levels than the apparent hypocalcemia group $(\mathrm{P}<0.001)$ (Table 1$)$.

Primary outcome. During a median follow-up duration of 25 (IQR, 8-53) months, 82 patients (29\%) reached the primary outcome. Thirty-four patients died and 48 were hospitalized for CVD. The Kaplan-Meier analysis showed that patients with low ionized calcium demonstrated a significantly higher likelihood of developing the primary outcome (log-rank $\mathrm{P}=0.01$ ) (Fig. $3 \mathrm{~A})$, while no significant difference was observed between patients with low and normal corrected calcium levels (Fig. 3B). The Cox proportional hazards model showed that low ionized calcium levels were significant risk factors for the primary outcome as compared to normal ionized calcium levels, regardless of the models employed (Table 2A). Even in Model 4 with all covariates included, the hazard ratio (HR) was 2.34 (95\% confidence interval [CI], 1.03-5.34; P=0.04). However, this was not the case when we used corrected calcium levels instead of ionized calcium levels to define hypocalcemia. The Kaplan-Meier analysis of the three groups (apparent hypocalcemia, hidden hypocalcemia, and normocalcemia) demonstrated that the prognosis of patients with hidden hypocalcemia was the worst in terms of the primary outcome (log-rank $\mathrm{P}=0.002$ ) (Fig. 3C). The univariate Cox proportional hazards model showed that hidden hypocalcemia was significantly associated with developing the primary outcome as compared to normocalcemia (HR, 2.51; 95\% CI, 1.41-4.47; P = 0.002) (Table 2B). After adjustment for other covariates, hidden hypocalcemia remained associated with a higher likelihood of developing the primary outcome in Model 4 (adjusted HR, 2.56; 


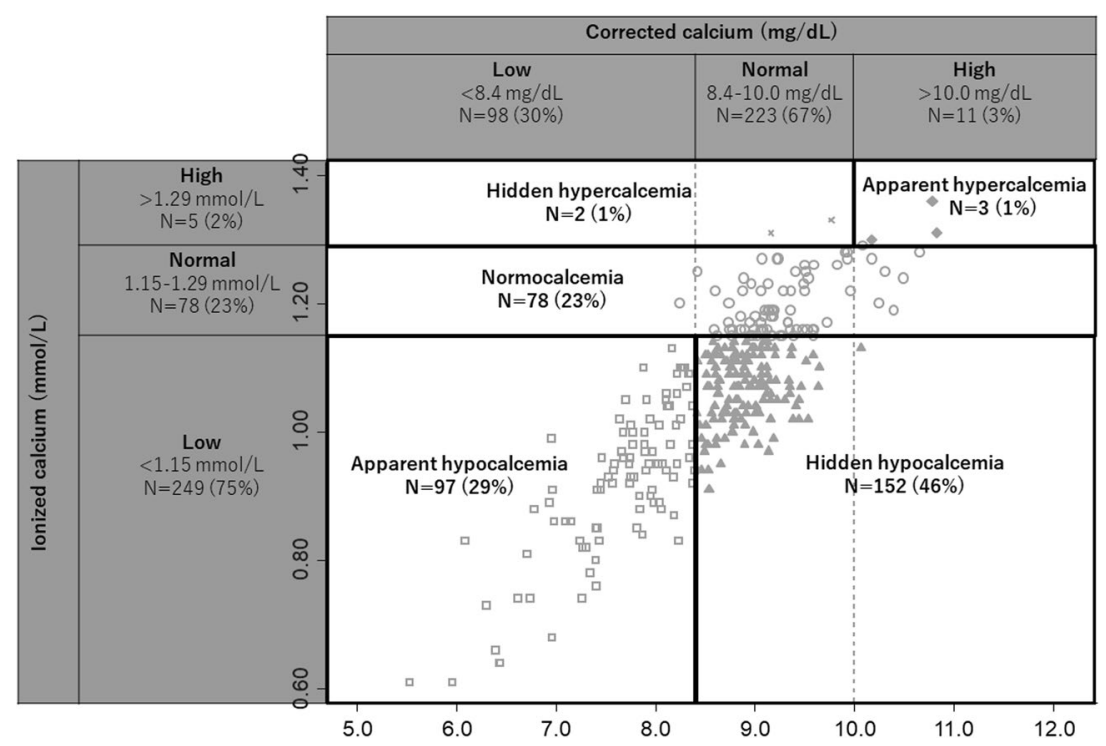

Figure 2. Scatter plot of ionized and corrected calcium at the initiation of dialysis. Apparent hypocalcemia, hidden hypocalcemia, and normocalcemia accounted for $29 \%, 46 \%$, and $23 \%$ of the enrolled patients, respectively. Most of the patients belonged to one of these three groups.

\begin{tabular}{|c|c|c|c|c|c|}
\hline Baseline characteristics & $\begin{array}{l}\text { Apparent } \\
\text { hypocalcemia } \mathrm{N}=97\end{array}$ & \begin{tabular}{|l|} 
Hidden \\
hypocalcemia \\
$\mathrm{N}=152$
\end{tabular} & $\begin{array}{l}\text { Normocalcemia } \\
N=78\end{array}$ & $\begin{array}{l}\text { Apparent vs. } \\
\text { Hidden }\end{array}$ & $\begin{array}{l}\text { ANOVA/ } \\
\text { Fisher's exact } \\
\text { test }\end{array}$ \\
\hline Ionized calcium $(\mathrm{mmol} / \mathrm{L})$ & $0.92 \pm 0.12$ & $1.07 \pm 0.05$ & $1.20 \pm 0.04$ & $<0.001$ & $<0.001$ \\
\hline Corrected calcium (mg/dL) & $7.6 \pm 0.6$ & $8.9 \pm 0.3$ & $9.3 \pm 0.5$ & $<0.001$ & $<0.001$ \\
\hline Age (years) & $61 \pm 15$ & $67 \pm 13$ & $62 \pm 16$ & 0.001 & 0.001 \\
\hline Male (\%) & 73 & 73 & 62 & 1.00 & 0.16 \\
\hline $\mathrm{eGFR}\left(\mathrm{ml} / \mathrm{min} / 1.73 \mathrm{~m}^{2}\right)$ & $4.7 \pm 1.6$ & $5.2 \pm 1.6$ & $5.4 \pm 2.3$ & 0.02 & 0.04 \\
\hline Body mass index & $24.5 \pm 4.5$ & $22.5 \pm 3.7$ & $23.1 \pm 6.5$ & $<0.001$ & 0.01 \\
\hline \multicolumn{6}{|l|}{ Comorbidities } \\
\hline Diabetes (\%) & 37 & 49 & 33 & 0.09 & 0.05 \\
\hline Coronary artery disease (\%) & 3 & 9 & 10 & 0.07 & 0.10 \\
\hline Heart failure (\%) & 4 & 11 & 14 & 0.09 & 0.06 \\
\hline Pacemaker implantation & 2 & 1 & 1 & 0.64 & 0.86 \\
\hline Peripheral artery disease (\%) & 6 & 3 & 6 & 0.35 & 0.41 \\
\hline Cerebrovascular infarction (\%) & 4 & 11 & 12 & 0.09 & 0.12 \\
\hline Phosphorus (mg/dL) & $6.6 \pm 1.9$ & $5.5 \pm 1.4$ & $5.2 \pm 1.6$ & $<0.001$ & $<0.001$ \\
\hline Alkaline phosphates (IU/L) & $249(177,305)$ & $219(179,298)$ & $222(171,270)$ & 0.62 & 0.18 \\
\hline Intact PTH $(\mathrm{pg} / \mathrm{mL})$ & $355(246,551)$ & $273(188,372)$ & $174(72,296)$ & $<0.001$ & $<0.001$ \\
\hline $\operatorname{Albumin}(\mathrm{g} / \mathrm{dL})$ & $3.1 \pm 0.6$ & $2.9 \pm 0.6$ & $3.3 \pm 0.5$ & 0.003 & $<0.001$ \\
\hline $\mathrm{CRP}(\mathrm{mg} / \mathrm{dL})$ & $0.17(0.05,0.82)$ & $0.12(0.02,0.89)$ & $0.06(0.02,0.21)$ & 0.55 & $<0.001$ \\
\hline $\mathrm{pH}$ & $7.37 \pm 0.05$ & $7.40 \pm 0.05$ & $7.38 \pm 0.05$ & $<0.001$ & $<0.001$ \\
\hline $\mathrm{HCO}^{-}(\mathrm{mmol} / \mathrm{L})$ & $20.2 \pm 4.1$ & $23.0 \pm 4.4$ & $21.5 \pm 4.4$ & $<0.001$ & $<0.001$ \\
\hline \multicolumn{6}{|l|}{ Prescription } \\
\hline VDRA (\%) & 25 & 32 & 46 & 0.26 & 0.01 \\
\hline Calcium bycarbonate (\%) & 63 & 41 & 62 & 0.001 & 0.001 \\
\hline
\end{tabular}

Table 1. Baseline characteristics in patients with apparent hypocalcemia, hidden hypocalcemia, and normocalcemia. Data are presented as mean $\pm \mathrm{SD}$, medians (interquartile ranges), or percentages. Group definitions: apparent hypocalcemia, low ionized calcium $(<1.15 \mathrm{mmol} / \mathrm{L})$, and low corrected calcium $(<8.4 \mathrm{mg} /$ $\mathrm{dL})$; hidden hypocalcemia, low ionized calcium $(<1.15 \mathrm{mmol} / \mathrm{L})$, and normal or high corrected calcium $(\geq 8.4 \mathrm{mg} /$ $\mathrm{dL}$ ); normocalcemia, normal ionized calcium (1.15-1.29 mmol/L). Abbreviations: eGFR, estimated glomerular filtration rate; PTH, parathyroid hormone; CRP, C-reactive protein; VDRA, vitamin D receptor activator.

95\% CI, 1.11-5.94; $\mathrm{P}=0.03$ ) (Table 2B). Apparent hypocalcemia was not a significant risk factor in univariate or multivariate Cox proportional hazards models (Table 2B). These results were similar in the analyses with normal ionized and corrected calcium as reference (Table 3 ). 
A

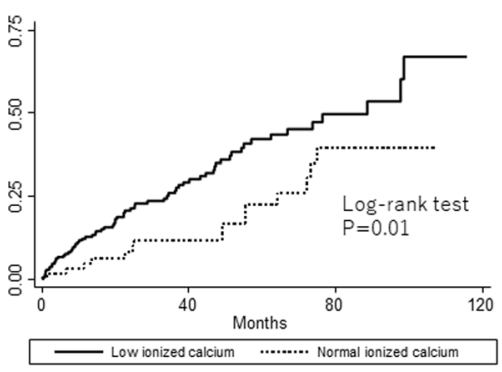

B

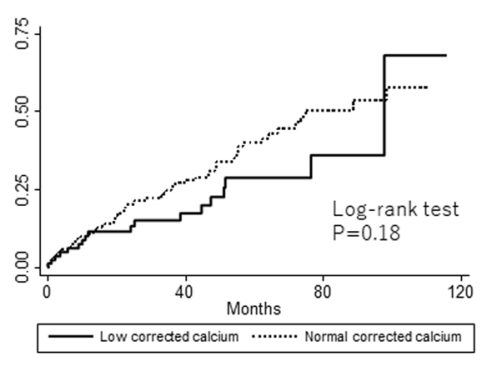

C

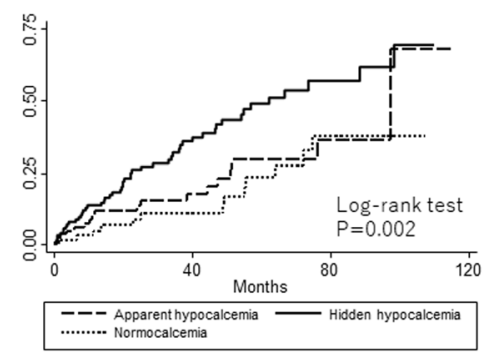

Figure 3. Kaplan-Meier failure function for time to all-cause death or hospitalization for cardiovascular disease in patients with low and normal ionized calcium (A), in patients with low and normal corrected calcium (B), and in patients with apparent hypocalcemia, hidden hypocalcemia, and normocalcemia (C).

\begin{tabular}{|c|c|c|c|c|c|c|}
\hline \multicolumn{7}{|l|}{ (A) } \\
\hline & \multicolumn{2}{|l|}{ Low corrected Ca } & \multirow{2}{*}{$\begin{array}{l}\text { Normal } \\
\text { corrected } \\
\mathrm{Ca}\end{array}$} & \multicolumn{2}{|l|}{ Low ionized Ca } & \multirow{2}{*}{$\begin{array}{l}\text { Normal } \\
\text { ionized Ca }\end{array}$} \\
\hline & Hazard ratio & P value & & Hazard ratio & $P$ value & \\
\hline Univariate & $0.70(0.41$ to 1.18$)$ & 0.18 & Reference & 2.04 (1.16 to 3.58$)$ & 0.01 & Reference \\
\hline Model 1 & $0.89(0.52$ to 1.51$)$ & 0.66 & Reference & $1.86(1.05$ to 3.26$)$ & 0.03 & Reference \\
\hline Model 2 & $0.93(0.53$ to 1.63$)$ & 0.81 & Reference & $2.36(1.30$ to 4.30$)$ & 0.01 & Reference \\
\hline Model 3 & $0.78(0.39$ to 1.56$)$ & 0.48 & Reference & $2.69(1.30$ to 5.58$)$ & 0.01 & Reference \\
\hline Model 4 & $0.88(0.42$ to 1.87$)$ & 0.74 & Reference & $2.34(1.03$ to 5.34$)$ & 0.04 & Reference \\
\hline \multicolumn{7}{|l|}{ (B) } \\
\hline & \multicolumn{2}{|l|}{ Apparent HypoCa } & & \multicolumn{2}{|l|}{ Hidden HypoCa } & \multirow{2}{*}{ NormoCa } \\
\hline & Hazard ratio & P value & & Hazard ratio & P value & \\
\hline Univariate & 1.34 (0.68 to 2.67$)$ & 0.40 & & 2.51 (1.41 to 4.47$)$ & 0.002 & Reference \\
\hline Model 1 & $1.50(0.75$ to 3.01$)$ & 0.25 & & 2.04 (1.13 to 3.68$)$ & 0.02 & Reference \\
\hline Model 2 & $1.86(0.90$ to 3.87$)$ & 0.10 & & $2.63(1.41$ to 4.90$)$ & 0.002 & Reference \\
\hline Model 3 & $1.81(0.72$ to 4.53$)$ & 0.21 & & $3.02(1.44$ to 6.34$)$ & 0.003 & Reference \\
\hline Model 4 & $1.79(0.65$ to 4.90$)$ & 0.26 & & $2.56(1.11$ to 5.94$)$ & 0.03 & Reference \\
\hline
\end{tabular}

Table 2. Associations of hypocalcemia defined by ionized or corrected calcium (A) and apparent hypocalcemia and hidden hypocalcemia (B) with the primary outcome. Model 1: univariate + age, sex, eGFR, and history of DM. Model 2: Model $1+$ past history of CVD. Model 3: Model $2+$ parameters of CKD-MBD and pH. Model 4: Model 3 + parameters of nutrition or inflammation. Abbreviations: eGFR, estimated glomerular filtration rate; $\mathrm{DM}$, diabetes mellitus; $\mathrm{CVD}$, cardiovascular disease; $\mathrm{CKD}-\mathrm{MBD}$, chronic kidney disease-mineral and bone disorder.

Intervention to hypocalcemia. Patients with hidden hypocalcemia were less likely to receive an intervention for hypocalcemia, such as increased dose of VDRA and use of calcium carbonate, than patients with apparent hypocalcemia (69\% vs. $83 \%$; Fisher's exact test, $\mathrm{P}=0.03$ ) (Fig. 4). Moreover, the percentage of the patients with both interventions were higher in the apparent hypocalcemia group than the hidden hypocalcemia group (39\% vs. $13 \%$; Fisher's exact test, $\mathrm{P}<0.001$ ). The Cox proportional hazards model only in the patients with low ionized calcium levels showed that any intervention to hypocalcemia was associated with a lower risk for the primary outcome in the univariate model $(\mathrm{HR}, 0.40 ; 95 \% \mathrm{CI}, 0.24-0.65 ; \mathrm{P}<0.001)$ and in the multivariate model (adjusted HR, 0.54; 95\% CI, 0.29-0.99; P = 0.047).

\section{Discussion}

In this retrospective cohort study, we revealed that true hypocalcemia (ionized calcium $<1.15 \mathrm{mmol} / \mathrm{L}$ ) accounted for $75 \%$ of the patients undergoing incident hemodialysis. Furthermore, $61 \%$ of the true hypocalcemia cases were found to be hidden hypocalcemia. We showed that true hypocalcemia was a risk factor for the composite of all-cause mortality and cardiovascular events, while hypocalcemia defined by corrected calcium was not. Moreover, hidden hypocalcemia proved to be a strong risk factor.

To the best of our knowledge, this study is the first study to demonstrate the high prevalence of true hypocalcemia and hidden hypocalcemia in pre-dialysis CKD stage 5. Regarding the prevalence of hypocalcemia defined by corrected calcium, our results were consistent with our previous study showing approximately $30 \%$ of patients in CKD stage 5 among a large CKD cohort ${ }^{19}$. The prevalence of hypocalcemia defined by ionized and corrected calcium levels in our cohort ( $75 \%$ and $30 \%$, respectively) was higher than that in the previous study $(32 \%$ and $16 \%$, respectively $)^{6}$. Furthermore, in our cohort, few patients showed true hypercalcemia, although $8.9 \%$ of the patients showed true hypercalcemia in the previous study ${ }^{6}$. The reason for this discrepancy might reside in the 


\begin{tabular}{|l|l|l|l|}
\hline \multicolumn{2}{|l|}{ Adjusted hazard ratio } & \multicolumn{2}{|l|}{ Corrected calcium } \\
\cline { 3 - 4 } & \multirow{3}{*}{ Ionized calcium } & Low & Normal \\
\hline \multirow{3}{*}{ Normal } & $\mathrm{N}=1$ & $\mathrm{~N}=64$ \\
\cline { 3 - 5 } & \multirow{2}{*}{ Low } & - & Reference \\
\hline & & $\mathrm{N}=83$ & $\mathrm{~N}=134$ \\
\cline { 3 - 4 } & $1.79(0.65-4.99)$ & $\mathbf{2 . 7 6 ( 1 . 1 6 - 6 . 5 9 )}$ \\
\hline
\end{tabular}

Table 3. Associations of calcium status stratified by ionized and corrected calcium with the primary outcome in the final model. Adjusted hazard ratios were estimated using model 4 with normal corrected and ionized calcium group as reference. Low ionized calcium levels despite normal corrected calcium levels (hidden hypocalcemia) was a significant risk for the primary outcome, while low ionized calcium levels and low corrected calcium levels (apparent hypocalcemia) was not. Since there was only 1 patient with normal ionized and low corrected calcium, adjusted hazard ratio in this group is not shown.

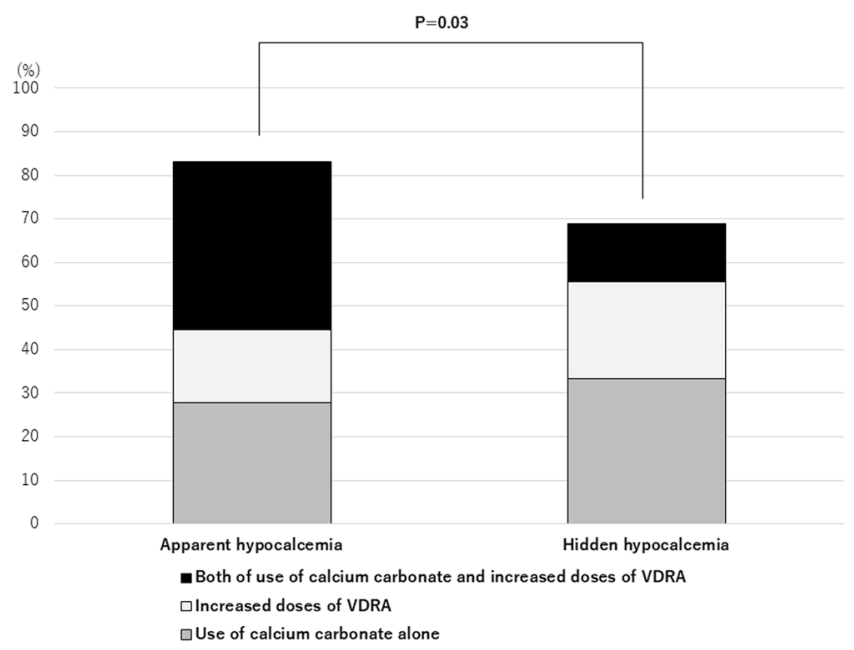

Figure 4. Percentage of patients who received intervention for hypocalcemia during hospitalization among patients with apparent hypocalcemia and hidden hypocalcemia. Intervention for hypocalcemia was defined as either increased doses of VDRA during hospitalization or calcium carbonate at discharge or both. Patients with hidden hypocalcemia were less likely to receive an intervention for hypocalcemia compared to patients with apparent hypocalcemia (Fisher's exact test; $\mathrm{p}<0.05$ ). Abbreviation: VDRA, vitamin D receptor activator.

different timings of blood sampling; calcium levels were measured just before the first dialysis in this study, while they were measured during the first 91 days of dialysis in the previous study. Corrected calcium levels increased after dialysis initiation among patients with low corrected calcium levels ${ }^{8}$, likely because of a positive calcium balance during dialysis ${ }^{20}$, active vitamin $\mathrm{D}$ treatment, and/or calcium-based phosphate binders. Despite the discrepancy, approximately $60 \%$ of the patients with true hypocalcemia were incorrectly categorized as normocalcemia using corrected calcium both in our cohort and in the previous study.

True hypocalcemia defined by low ionized calcium is a significant risk factor for mortality or CVD morbidity. Our results agree with those of a previous report from the Dialysis Outcomes and Practice Pattern Study (DOPPS) showing worse prognosis of hypocalcemic patients ${ }^{21}$. In this previous study, both uncorrected calcium and corrected calcium levels of $7.5 \mathrm{mg} / \mathrm{dL}$ or less were associated with a greater mortality in patients with albumin levels higher than $3.8 \mathrm{~g} / \mathrm{dL}$, i.e. a subpopulation in whom the correction of calcium was almost unnecessary to infer ionized calcium. Causal relationship between low ionized calcium and the outcome, if any, includes the following issues. Hypocalcemia leads to heart failure ${ }^{22,23}$ and arrhythmia ${ }^{24}$. Moreover, in patients undergoing hemodialysis, hypocalcemia is more likely to be associated with a positive net balance of calcium during dialysis ${ }^{19}$. Positive calcium balance is a risk of myocardial infarction, especially in diabetic patients with low $\mathrm{PTH}^{25}$. This might be explained by exacerbation of vascular calcification. Another possibility is residual confounding by poor nutritional status, since patients with low ionized calcium tended to have older age, lower BMI, lower albumin levels, and diabetes. In fact, we adjusted for these covariates when studying the association between low ionized calcium levels and the outcome. However, malnutrition cannot be sufficiently explained only by these traditional markers.

Prior studies reported the association between higher corrected calcium and poor prognosis ${ }^{8,9}$. This association might be partly explained by overestimation of calcium status among patients with hypoalbuminemia, who are at high risk for mortality ${ }^{26}$. Since albumin is an important carrier for calcium ${ }^{1}$, uncorrected calcium levels are intrinsically dependent on serum albumin levels. In fact, in the aforementioned study ${ }^{21}$, uncorrected calcium 
levels of $7.5 \mathrm{mg} / \mathrm{dL}$ or less were not high risk for mortality in hypoalbuminemic patients (less than $3.8 \mathrm{~g} / \mathrm{dL}$ ). In other words, serum albumin levels modify the association between uncorrected calcium and mortality.

However, we should not forget that the equation of corrected calcium cannot be used to infer ionized calcium levels just before the dialysis initiation, when discussing the association between calcium status and prognosis. Although true hypocalcemia defined by low ionized calcium was a significant risk factor, hypocalcemia defined by low corrected calcium was not. Misclassifications of low calcium status possibly result in an overestimation of the prognosis. In other words, physicians may overlook the worse prognosis of patients with hidden hypocalcemia on the grounds of their normal corrected calcium levels.

Notably, hidden hypocalcemia, and not apparent hypocalcemia, was associated with a higher likelihood of developing the primary outcome, although ionized calcium levels were much lower in the apparent hypocalcemia group than in the hidden hypocalcemia group. Patients with hidden hypocalcemia were more likely to have a history of diabetes, PCI/CABG, and heart failure than patients with apparent hypocalcemia. Additionally, patients with hidden hypocalcemia had higher serum bicarbonate levels $(\mathrm{pH})$ than patients with other calcium statuses, suggesting lower protein intake in this population ${ }^{27}$. Low protein intake accelerates body weight loss ${ }^{28}$. In fact, patients with hidden hypocalcemia had lower BMI and serum albumin levels than patients with apparent hypocalcemia. Since, the definition of cachexia includes weight loss and low serum albumin ${ }^{28}$, patients with hidden hypocalcemia might have suffered from cachexia, which is a complex metabolic syndrome associated with underling chronic illnesses such as CKD or chronic heart failure ${ }^{28}$. Patients with hidden hypocalcemia were likely to have abnormal high ALP despite a lower iPTH than patients with apparent hypocalcemia. High ALP levels relative to PTH is characteristic of osteomalacia ${ }^{29}$, possibly derived from vitamin D deficiency ${ }^{30}$. Weight loss and vitamin $\mathrm{D}$ deficiency, which suggest malnutrition, are risk factors for mortality in patients undergoing hemodialysis ${ }^{31-33}$. However, hidden hypocalcemia remained a significant risk factor for the primary outcome after adjustment for a history of diabetes, PCI/CABG and heart failure, ALP, iPTH, pH, BMI, and albumin. Therefore, hidden hypocalcemia might reflect malnutrition status, which cannot be sufficiently explained by these standard nutritional parameters.

Patients with hidden hypocalcemia were less likely to receive VDRA or calcium carbonate than patients with apparent hypocalcemia, because physicians may miss true hypocalcemia. Physicians can readily recognize hypocalcemia in patients with apparent hypocalcemia but cannot recognize hidden hypocalcemia unless they check ionized calcium levels. In our study, the data of ionized calcium were extracted from the blood gas test, which was performed for evaluating $\mathrm{pH}$ or bicarbonate $\left(\mathrm{tCO}_{2}\right)$. Since the data of ionized calcium were "byproducts," physicians may not check that data. Moreover, patients with apparent hypocalcemia were more likely to receive both calcium carbonate and an increased dose of VDRA than patients with hidden hypocalcemia. This practice pattern suggests a strong intention of physicians to increase serum calcium levels in hypocalcemic patients. Intervention to treat hypocalcemia, such as the administration of VDRA and calcium carbonate, improves hypocalcemic cardiomyopathy ${ }^{34,35}$. We found that VDRA or calcium carbonate prescription was associated with lower CVD morbidity and mortality among hypocalcemic patients. Previous observational studies showed that the use of VDRA was associated with a better prognosis among dialysis patients ${ }^{33,36-39}$. Furthermore, Inaguma et al. reported that the use of calcium carbonate before the initiation of dialysis was associated with better prognosis after the initiation of dialysis ${ }^{9}$. In this context, undertreatment for hypocalcemia might explain the observed higher risk in patients with hidden hypocalcemia. Our observation raises a question about the revised Kidney Disease: Improving Global Outcomes guidelines on CKD-MBD ${ }^{40}$, arguing that asymptomatic or mild hypocalcemia does not need to be corrected considering the unproven benefits of intervention to hypocalcemia and potential harm of a positive calcium balance ${ }^{41,42}$.

Our study has several strengths. First, we measured the ionized calcium levels immediately after drawing the samples, which is in sharp contrast to the previous study ${ }^{6}$. Ionized calcium levels vary easily due to $\mathrm{CO}_{2}$ changes in the samples ${ }^{43,44}$. Fresh samples are required to measure these levels accurately. Second, this is the first study to measure the ionized calcium levels immediately before the initiation of dialysis, partly reflecting a patient's nutritional status and pre-dialysis care not influenced by hemodialysis.

Our study has several limitations. First, a single-center study limits generalizability to other populations. Second, the outcomes were not adjudicated. Indication of hospitalization possibly varies according to the physicians. Further multicenter studies with a larger number of patients are needed to validate the association between calcium status and hard outcome.

\section{Conclusion}

Hidden hypocalcemia at the initiation of dialysis was a strong risk factor for the composite outcome of all-cause mortality and CVD morbidity. This suggests the importance of measuring ionized calcium levels in patients undergoing incident hemodialysis.

Received: 31 October 2019; Accepted: 26 February 2020;

Published online: 10 March 2020

\section{References}

1. Anonymous. Correcting the calcium. British Medical Journal. 1, 598 (1977).

2. Clase, C. M., Norman, G. L., Beecroft, M. L. \& Churchill, D. N. Albumin-corrected calcium and ionized calcium in stable haemodialysis patients. Nephrol. Dial. Transplant. 15, 1841-1846 (2000).

3. Jain, A., Bhayana, S., Vlasschaert, M. \& House, A. A formula to predict corrected calcium in haemodialysis patients. Nephrol. Dial. Transplant. 23, 2884-2888 (2008).

4. Gauci, C. et al. Pitfalls of measuring total blood calcium in patients with CKD. J. Am. Soc. Nephrol. 19, 1592-1598 (2008).

5. Anonymous. Comorbid conditions and correlations with mortality risk among 3,399 incident hemodialysis patients. Am. J. Kidney Dis. 20, 32-38 (1992). 
6. Obi, Y. et al. Hidden Hypercalcemia and Mortality Risk in Incident Hemodialysis Patients. J. Clin. Endocrinol. Metab. 101, 2440-2449 (2016).

7. Rivara, M. B. et al. Uncorrected and Albumin-Corrected Calcium, Phosphorus, and Mortality in Patients Undergoing Maintenance Dialysis. J. Am. Soc. Nephrol. 26, 1671-1681 (2015).

8. Obi, Y. et al. Association of Pre-ESRD Serum Calcium with Post-ESRD Mortality Among Incident ESRD Patients: a Cohort Study. J. Bone Miner. Res. 33, 1027-1036 (2018).

9. Inaguma, D. et al. Relationship between serum calcium level at dialysis initiation and subsequent prognosis. Renal Replacement Therapy. 3, (2017).

10. Lu, J. L. et al. Racial Differences in Association of Serum Calcium with Mortality and Incident Cardio- and Cerebrovascular Events. J. Clin. Endocrinol. Metab. 101, 4851-4859 (2016).

11. Miura, S. et al. Association of Hypocalcemia With Mortality in Hospitalized Patients With Heart Failure and Chronic Kidney Disease. J. Card. Fail. 21, 621-627 (2015).

12. Sumida, K. et al. Prognostic significance of pre-end-stage renal disease serum alkaline phosphatase for post-end-stage renal disease mortality in late-stage chronic kidney disease patients transitioning to dialysis. Nephrol. Dial. Transplant 33, 264-273 (2018).

13. Rhee, C. M. et al. Pre-dialysis serum sodium and mortality in a national incident hemodialysis cohort. Nephrol. Dial. Transplant. 31, 992-1001 (2016)

14. Kovesdy, C. P. et al. Abrupt Decline in Kidney Function Precipitating Initiation of Chronic Renal Replacement Therapy. Kidney Int. Reports 3, 602-609 (2017).

15. Kleine, C. E. et, al. Association of Pre-End-Stage Renal Disease Hemoglobin with Early Dialysis Outcomes. Am. J. Nephrol. 47, $333-342$ (2018)

16. KDIGO. Clinical Practice Guideline for the Diagnosis, Evaluation, Prevention, and Treatment of Chronic Kidney Disease-Mineral and Bone Disorder (CKD-MBD). Kidney Int. 76, S1-130 (2009).

17. Fukagawa, M. et al. Clinical practice guideline for the management of chronic kidney disease-mineral and bone disorder. Ther. Apher. Dial. 17, 247-88 (2013).

18. Siggaard-Anderson, O., Thode, J., \& Wandrup, JH. The concetration of free calcium ions in the blood plasma ionized calcium. In: Siggaard-Anderson, ed. Proceedings of the IFCC expert panel on pH and blood gases held at Herlev Hospital 1980. Copenhagen: Radiometer Medical A/S, 163-190 (1981).

19. Oka, T. et al. Proteinuria-associated renal magnesium wasting leads to hypomagnesemia: a common electrolyte abnormality in chronic kidney disease. Nephrol. Dial. Transplant. 1, 1154-1162 (2018).

20. Drüeke, T. B. \& Touam, M. Calcium balance in haemodialysis-do not lower the dialysate calcium concentration too much (con part). Nephrol. Dial. Transplant. 24, 2990-2993 (2009).

21. Tentori, F. et al. Mortality Risk for Dialysis Patients With Different Levels of Serum Calcium, Phosphorus, and PTH: The Dialysis Outcomes and Practice Patterns Study (DOPPS). Am. J. Kidney Dis. 52, 519-530 (2008).

22. Connor, T. B., Rosen, B. L., Blaustein, M. P., Applefeld, M. M. \& Doyle, L. A. Hypocalcemia Precipitating Congestive Heart Failure. N. Engl. J. Med. 307, 869-872 (1982).

23. Newman, D. B. et al. Reversible cardiac dysfunction associated with hypocalcemia: a systematic review and meta-analysis of individual patient data. Heart Fail. Rev. 19, 199-205 (2014).

24. Mozos, I. Laboratory markers of ventricular arrhythmia risk in renal failure. Biomed Res. Int. 2014, 509204 (2014).

25. Tagawa, M., Hamano, T., Sueta, S., Ogata, S. \& Saito, Y. Higher dialysate calcium concentration is associated with incident myocardial infarction among diabetic patients with low bone turnover: a longitudinal study. Sci. Rep. 8, 10060 (2018).

26. Zitt, E. et al. Interaction of time-varying albumin and phophorus on mortality in incident dialysis patietns. Clin. J. Am. Soc. Nephorl. 11, 2650-2656 (2011).

27. Frassetto, L. A., Todd, K. M., Morris, R. C. Jr. \& Sebastian, A. Estimation of net endogenous noncarbonic acid production in humans from diet potassium and protein contents. Am. J. Clin. Nutr. 68, 576-83 (1998).

28. Evans, W. J. et al. Cachexia: A new definition. Clin. Nutr. 27, 793-799 (2008).

29. Coen, G. et al. Renal bone disease in 76 patients with varying degrees of predialysis chronic renal failure: a cross-sectional study. Nephrol. Dial. Transplant 11, 813-819 (1996).

30. Mac-Way, F., Azzouz, L., Noel, C. \& Lafage-Proust, M.-H. Osteomalacia induced by vitamin D deficiency in hemodialysis patients: the crucial role of vitamin D correction. J. Bone Miner. Metab. 32, 215-219 (2014).

31. Kalantar-Zadeh, K. \& Kopple, J. D. Obesity paradox in patients on maintenance dialysis. Contrib. Nephrol. 151, 57-69 (2006).

32. Pilz, S., Iodice, S., Zittermann, A., Grant, W. B. \& Gandini, S. Vitamin D status and mortality risk in CKD: a meta-analysis of prospective studies. Am. J. Kidney Dis. 58, 374-382 (2011).

33. Wolf, M. et al. Vitamin D levels and early mortality among incident hemodialysis patients. Kidney Int. 72, 1004-1013 (2007).

34. Venugopalan, G. et al. Hypocalcemic Cardiomyopathy Due to Vitamin D Deficiency in a Very Old Man. Journal of the American Geriatrics Society. 8, 1708-1709 (2015).

35. Kazmi, A. S. \& Wall, B. M. Reversible congestive heart failure related to profound hypocalcemia secondary to hypoparathyroidism. Am. J. Med. Sci. 333, 226-229 (2007).

36. Shoji, T. et al. Lower risk for cardiovascular mortality in oral 1alpha-hydroxy vitamin D3 users in a haemodialysis population. Nephrol. Dial. Transplant 19, 179-184 (2004).

37. Kalantar-Zadeh, K. et al. Survival predictability of time-varying indicators of bone disease in maintenance hemodialysis patients. Kidney Int. 70, 771-780 (2006).

38. Wolf, M. et al. Impact of activated vitamin D and race on survival among hemodialysis patients. J. Am. Soc. Nephrol. 19, 1379-1388 (2008).

39. Naves-Diaz, M. et al. Oral active vitamin D is associated with improved survival in hemodialysis patients. Kidney Int. 74, 1070-1078 (2008).

40. Kidney Disease. Improving Global Outcomes (KDIGO) CKD-MBD Update Work Group, Disease, K. \& Global Outcomes CKDMBD Update Work Group, I. KDIGO 2017 Clinical Practice Guideline Update for the Diagnosis, Evaluation, Prevention, and Treatment of Chronic Kidney Disease-Mineral and Bone Disorder (CKD-MBD). Kidney International Supplements. 7, 1-59 (2017).

41. Spiegel, D. M. \& Brady, K. Calcium balance in normal individuals and in patients with chronic kidney disease on low- and highcalcium diets. Kidney Int. 81, 1116-1122 (2012).

42. Raggi, P., Bommer, J. \& Chertow, G. M. Valvular calcification in hemodialysis patients randomized to calcium-based phosphorus binders or sevelamer. J. Heart Valve Dis. 13, 134-141 (2004).

43. Siyam, F. F. \& Klachko, D. M. What is hypercalcemia? The importance of fasting samples. Cardiorenal Med. 3, 232-238 (2013).

44. Baird, G. S. Ionized calcium. Clin. Chim. Acta 412, 696-701 (2011).

\section{Acknowledgements}

We thank Dr. Masatou Tanaka (Tanaka Urology Clinic Dialysis Center Furuedai), Dr. Eikun Maruyama (Kitagawa Clinic), Dr. Tsujimoto Yoshihiro (Inoue Hospital), Dr. Hideyuki Nakagawa (Nakagawa Clinic), Dr. Hiroko Tokizane (Tokizane Clinic), Dr. Shigeru Hanada (Taisei Clinic), Dr. Kazuhisa Yamamoto (Sanko Hospital), Dr. Hironori Kawamura (Kawamura Clinic), Dr. Jun Matsuda (Suita City Hospital), Dr. Ken Takahara 
(Sone Hospital), Dr. Takahide Sugiyama (Tsukuno Fujii Clinic), Dr. Masashi Shinozaki (Nakano Clinic), Dr. Mutsuko Mori (Mori Clinic), Dr. Yokokawa Tomoko (Itami Gardens Clinic), Dr. Hiroshi Miyao (Miyao Urology Clinic), Dr. Yutaka Matsui (Matsui Dietary \& Dementia Clinic), Dr. Ken Kishida (Kishida Clinic), Dr. Takeshi Omichi (Omichi Clinic), Dr. Takehisa Hotta (Iseikai Fuzoku Clinic), Dr. Zenzo Fujii (St. Hill Hospital), Dr. Jong II Kim (Chibune Kidney and Dialysis Clinic), Dr. K.Y. (Tanaka Clinic), Dr. Shunro Agata (Nagahara Hospital), Dr. Shintaro Yamanaka (Shingu Municipal Medical Center), Dr. Yukito Kokado (Takahashi Clinic), Dr. Toshiaki Hirabayashi (Heimeikai Clinic), Dr. Takahiro Fujii (Itami Bara Clinic), Dr. Yutaro Azuma (Azuma Dialysis Clinic), Dr. Nobuhiko Narukawa (Sakuragaoka Hospital), Dr. Noriko Mikami (Mikami Clinic), Dr. Mitsuru Kubo (Kyowakai Hospital), Dr. Akira Okasho (Okasho Urology Clinic), Dr. Motohiro Okamura (Okamuraiin Jin Clinic), Dr. Hiroyuki Araki (Settsu Iseikai Hospital), Dr. Akira Wada (Azuma Renal Clinic), Dr. Masugi Satoh (Satoh Clinic Kuzuha), Dr. Yasuhiro Enomoto (Enomoto Clinic), Dr. Hidekazu Shimizu (Onoyama Clinic), Dr. Kazuyuki Hayashi (Ikeda Municipal Hospital), Dr. Keiji Isshiki (Tomita Clinic), Dr. Tohru Takahashi (Takahashi Clinic), Dr. Daisuke Nakagawa (Second Rokushima Clinic), Dr. Kiyoshi Shozu (Aino Hospital), Dr. Yumiko Honda (Toyonakamidorigaoka Hospital), Dr. Kei Hirai (Sanko Clinic), Dr. Toru Yoshie (Yoshie Hospital), Dr. Isao Fukuhara (Heisei Urologic Clinic), Dr. Sachiko Yamada (Saiseikai Ibaraki Hospital), Dr. Takanori Nagai (Nagai Clinic), Dr. Takeshi Tomokuni (Ibaraki Iseikai Hospital), Dr. Toyofumi Fukuda (Ibuki Clinic), Dr. Kanji Yoshida (Suzawa Clinic), Dr. Fumi Sato (Otemae Hospital), Dr. Kiyoshi Goto (Ono-naika Clinic), Dr. Ryoichi Miyazaki (Fujita Memorial Hospital), Dr. Yoshiaki Hirosue (Takasago Municipal Hospital), Dr. Chikako Monden (Kisei Hospital), Dr. Masato Baden (Takarazuka Hospital), Dr. Jyunya Takahara (Takahara Clinic), Dr. Jun Tomita (Toyonaka Watanabe Clinic, Kansai Medical Hospital), and Dr. Akira Moriguchi (Umeda-Higashi Blood Purification Clinic) for their assistance in collecting data. This study has been performed without the support of Grant. Funders/Sponsors had no role in analysis and interpretation of the data; preparation, review, or approval of the manuscript; and decision to submit the manuscript for publication.

\section{Author contributions}

The authors' responsibilities were as follows-S.Yamaguchi, T.H., Y.D., T.O., Y.S., and Y.I. contributed to the study design. S.Yamaguchi, T.H., Y.D., T.O., S.K., K.K., S.Yasuda, K.S., A.M., N.H., Y.S., I.M. and Y.I. were involved in data acquisition. Data analysis was done by S.Yamaguchi. Supervision or mentorship was done by T.H. and Y.I. All authors contributed to interpretation of the results. S.Yamaguchi and T.H. drafted the report, and the other authors gave critical revision of the manuscript for scientific and factual content. All authors read and approved the final manuscript.

\section{Competing interests}

S. Yamaguchi have received honoraria from Chugai Pharmaceutical Co., Ltd. T.H. and Y.S. have received honoraria from Chugai Pharmaceutical Co., Ltd., Kyowa Kirin Co., Ltd., and Fuso Pharmaceutical Industries, Ltd. and research grant support from Chugai Pharmaceutical Co., Ltd., and Fuso Pharmaceutical Industries. Y.I. have received honoraria from Chugai Pharmaceutical Co., Ltd., Kyowa Kirin Co., Ltd., and Teijin Pharma Ltd. All other authors declare no conflicts of interest.

\section{Additional information}

Correspondence and requests for materials should be addressed to T.H.

Reprints and permissions information is available at www.nature.com/reprints.

Publisher's note Springer Nature remains neutral with regard to jurisdictional claims in published maps and institutional affiliations.

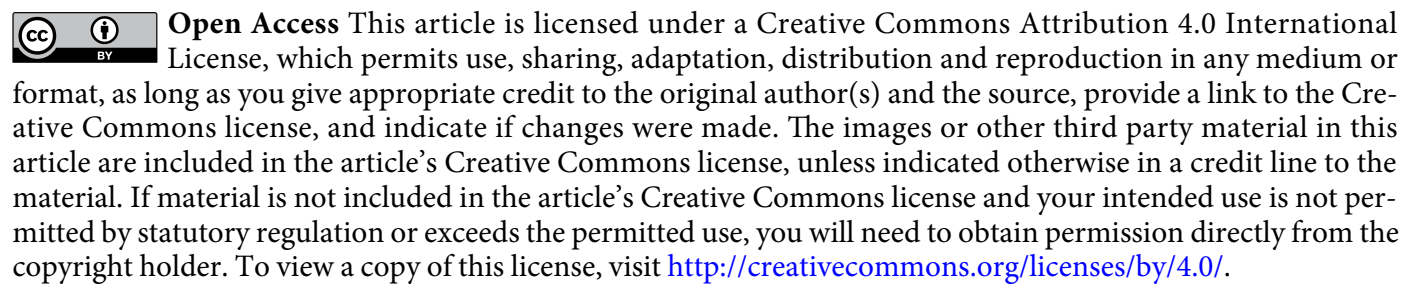

(c) The Author(s) 2020 